\title{
CLE and BAM genes in phloem development in potato (Solanum tuberosum L.)
}

Losev M.R. ${ }^{1 *}$, Gancheva M.S. ${ }^{1,2}$, Poliushkevich L.O. ${ }^{1}$, Lutova L.A. ${ }^{1}$

${ }^{1}$ Saint-Petersburg State University, St. Petersburg, Russia

${ }^{2}$ All-Russia Research Institute for Agricultural Microbiology, St. Petersburg, Russia

*email: st069730@student.spbu.ru

The phloem is one of the vascular tissues in plants that transports sugars, as well as other important metabolites such as phytohormones and messenger RNAs. It is shown that tuber formation is associated with increased phloem development in the forming tubers, which promotes the influx of substances into the developing tubers. The object of our research is potato, which is an important agricultural crop. The control of phloem development in potato is currently poorly understood, but there is evidence that CLE peptide hormones may be involved in this process. So, in Arabidopsis thaliana, the AtCLE25 peptide is a positive phloem regulator, but AtCLE45 is a negative one. Their receptors are members of the leucine-rich repeat receptor kinase family (CLERK-CLV2 and BAM-CLV1/CLV2 respectively). However, the role of the signal pathway triggered by CLE peptides in phloem development has not been previously studied in potato. In the data on differential gene expression in the potato phloem, we found an accumulation of transcripts of the StCLE19, StCLE12, StBAM1-2, StCLV1, and StBAM3-1 genes. When comparing the sequences of CLE peptides in potato and in Arabidopsis thaliana, it was found that StCLE19 differs from AtCLE25 by only one amino acid, which made the StCLE19 peptide a candidate for the role of a key player in the development of phloem in potato. In turn, the StCLE12 peptide is identical to the AtCLE41 and AtCLE44 peptides, which are involved in the regulation of the activity of another tissue - the cambium. We created constructs for the overexpression of the StCLE12 and StCLE19 genes and found that StCLE12 is indeed involved in the regulation of cambium cell division, and StCLE19 is involved in the development of the phloem. We also created constructs for analyzing the activity of promoters of the StBAM1-2, StCLV1, and StBAM3-1 genes.

Acknowledgements: This work was supported by the RFBR grant 20-316-80004. 